\title{
An on-line solid phase extraction procedure for the routine quantification of urinary methylmalonic acid by liquid chromatography-tandem mass spectrometry
}

\author{
Fabian Kirchhoff ${ }^{1, *}$, Stephan Lorenzl ${ }^{2}$ and \\ Michael Vogeser ${ }^{1}$ \\ ${ }^{1}$ Institute of Clinical Chemistry, Hospital of the University \\ of Munich, Munich, Germany \\ ${ }^{2}$ Clinic of Neurology, Hospital of the University of \\ Munich, Munich, Germany
}

\begin{abstract}
Background: The goal of this study was to develop and to validate an improved isotope-dilution-liquid chromatography-tandem mass spectrometry (LC-MS/MS) method for the quantification of methylmalonic acid (MMA) in urine.

Methods: A previously described sample preparation protocol requires two solvent extraction steps, including evaporation. The first extraction is to extract the analyte from the sample, and second occurs following derivatization of the extract. In the method described here, the second evaporation step was substituted by on-line solid phase extraction employing column-switching and a permanent co-polymer based extraction cartridge. A standard validation protocol was applied to investigate the performance of the method.

Results: The method was found to be linear in the clinically relevant range of concentrations $(6-100 \mu \mathrm{mol} / \mathrm{L})$. Total coefficients of variation were below $10 \%$ and inaccuracy was $<10 \%$ for quality control samples at three concentrations. Conclusions: By omitting one evaporation step, the semiautomated method described in this article enables for more convenient work-flow in the quantification of urinary MMA compared to the previous protocol. This is of relevance for MMA measurement in the routine clinical laboratory setting. Validation demonstrated acceptable analytical performance. Clin Chem Lab Med 2010;48:1647-50.
\end{abstract}

Keywords: liquid chromatography-tandem mass spectrometry (LC-MS/MS); methylmalonic acid (MMA); on-line solid phase extraction (SPE); urine.

\footnotetext{
*Corresponding author: Fabian Kirchhoff, Institute of Clinical Chemistry, Hospital of the University of Munich, Marchioninistraße 15, 81377 Munich, Germany Phone: +49 897095 3248, Fax: +49 897095 6220, E-mail: Fabian.Kirchhoff@med.uni-muenchen.de Received December 23, 2009; accepted May 21, 2010; previously published online August 13, 2010
}

Increased concentrations of methylmalonic acid (MMA) are recognized as a useful tool for the detection of cobalamin (vitamin B12) deficiency at the tissue level (1-3). Whereas serum cobalamin concentrations can be determined using high throughput ligand binding methods, reliable quantification of MMA in biological matrices requires the use of mass spectrometric methods. Since the degree of automation in mass spectrometric assays is limited (4), MMA quantification is not used at present for screening of cobalamin deficiency. Rather, it is typically used as a confirmatory second line test in cases where vitamin B12 concentrations are below $300 \mathrm{pg} / \mathrm{mL}$.

The assessment of MMA concentrations in serum is hampered by the fact that a decreased glomerular filtration rate is associated with increased serum MMA concentrations, irrespective of the cobalamin status $(5,6)$. In contrast, quantification of the urinary MMA/creatinine-ratio is not subject to interference by impaired renal function, and is thus superior to serum MMA measurements (7). Furthermore, urine MMA concentrations are about 100-fold higher compared to serum, which favours the robustness of analytical methods for urine as the sample material (reference ranges (8): serum MMA, $<0.44 \mu \mathrm{mol} / \mathrm{L}$ [conversion of units for methylmalonic acid: $(\mu \mathrm{mol} / \mathrm{L}) \times 0.118=(\mathrm{mg} / \mathrm{L})]$; urinary $\mathrm{MMA} /$ creatinine-ratio, $<3.56 \mathrm{mmol} / \mathrm{mol}$; assuming a typical mean urinary creatinine concentration of roughly $8.5 \mathrm{mmol} / \mathrm{L}$, urinary MMA concentrations $<30 \mu \mathrm{mol} / \mathrm{L}$ can be considered normal).

Gas chromatography-mass spectrometry (GC-MS) methods for the quantification of urinary MMA were introduced in the late 1970s (9). To date, two liquid chromatographytandem mass spectrometry (LC-MS/MS) methods for quantification of urinary MMA have been described $(10,11)$. Due to the highly-specific detection principle, the need for time consuming chromatographic separation is, in general, minimized in LC-MS/MS and many laboratories are now trying to switch MMA quantification from GC-MS to LC-MS/MS.

The methods that have been described for quantification of urinary MMA using LC-MS/MS include a multi-step sample preparation protocol. While the method described by Magera et al. (11) uses solid phase extraction, the method by Kushnir et al. (10) employs the more economic principle of solvent extraction. This protocol includes a solvent extraction step with evaporation of the extracts both before and after the derivatization step. The aim of our work was to 
improve this previously reported process of sample preparation for quantification of urinary MMA using LC-MS/MS by applying on-line solid phase extraction (SPE) with a permanent extraction cartridge (a scheme of the on-line SPE procedure is shown in Figure 1A).

We used a Waters Alliance 2795 HPLC module (Waters, Milford, MA, USA), coupled splitless to a Micromass Quattro LC tandem mass spectrometry system (Waters, Manchester, UK). For on-line solid phase extraction, a SPE column was used, together with a six-port high-pressure switching valve. Mass spectrometric conditions are listed in the online data supplement, Appendix 1.

Calibrators were made by diluting a stock solution of MMA with water to obtain MMA concentrations of 101.6, $76.2,50.8,38.1,25.4,12.7,6.4 \mu \mathrm{mol} / \mathrm{L}$. We used three levels of quality control samples, prepared by spiking pooled urine from healthy volunteers. Before spiking, the MMA concentration of this pool was determined by an independent GC-MS method, with cross-validation by an external laboratory. Target concentrations of the three quality control samples were calculated from the concentration of the pool and from the amount of spiked MMA. Aliquots of the quality control samples, as well as calibrators were stored at $-20^{\circ} \mathrm{C}$.

Two urine pools were prepared for additional validation of reproducibility in the use of the method in an epidemiological study.

The complete sample preparation and on-line SPE procedure is provided in the legend to Figure 1. The retention time of dibutyl-MMA and the internal standard dibutyl- $\mathrm{d}_{3}$-MMA was approximately $4.9 \mathrm{~min}$ after injection onto the extraction column. The total analytical run time was $6.5 \mathrm{~min}$. For quantification, the peak area-ratios of the multiple reaction mon-
A

a

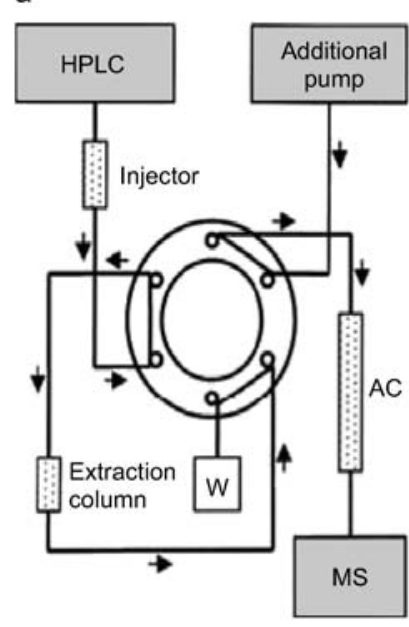

B

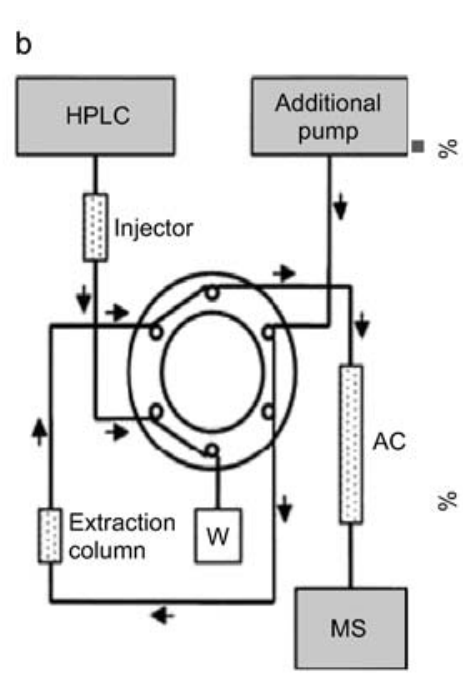

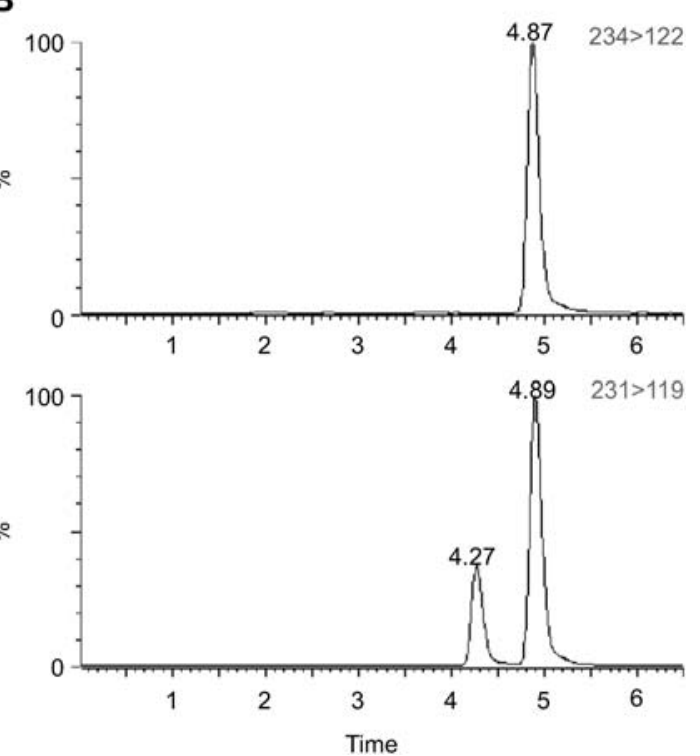

Figure 1 (A) Scheme of the on-line solid phase extraction system (AC, analytical column; W, waste; MS, mass spectrometry system).

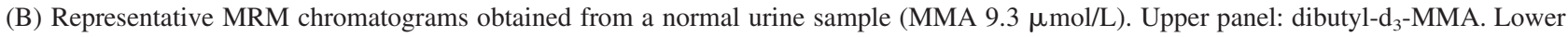
panel: dibutyl-MMA (retention time approx. $4.9 \mathrm{~min}$ ) base-line separated from dibutyl-SA (retention time approx. $4.3 \mathrm{~min}$ ).

Sample preparation protocol: Into $1.5 \mathrm{~mL}$ polypropylene cups, $50 \mu \mathrm{L}$ of calibrator, quality control samples or human urine samples was mixed with $50 \mu \mathrm{L}$ of $\mathrm{d}_{3}$-MMA $(8.32 \mu \mathrm{mol} / \mathrm{L})$ and $25 \mu \mathrm{L}$ of $1 \mathrm{M} \mathrm{HCl}$. After equilibration, $1600 \mu \mathrm{L}$ methyl tertiary butyl ether (MTBE) was added and the cups were shaken for $10 \mathrm{~min}$ using a horizontal shaker. After stopping the shaking, a complete separation between aqueous and organic phase was obtained within seconds without centrifugation, with the organic layer above the aqueous phase. The organic phase was transferred into a $2.0 \mathrm{~mL}$ polypropylene cup, which was then placed into an evaporation device, thermostated at $37^{\circ} \mathrm{C}(\mathrm{Barkey}$ Vapotherm, Labortechnik Barkey, Leopoldsheim, Germany). The extract was evaporated to dryness under a flow of nitrogen. The residue was dissolved in $50 \mu \mathrm{L}$ of the derivatization reagent $3 \mathrm{~N} \mathrm{HCl} / \mathrm{BuOH}$ by vortex mixing. The cups were opened and placed uncapped into

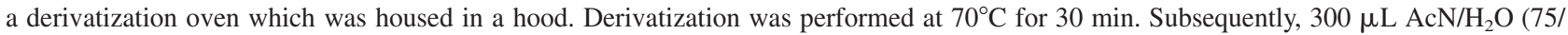
25 by volume) was added to the tubes. After mixing, $100 \mu \mathrm{L}$ of this solution were transferred into HPLC vials. On-line solid phase extraction protocol: A Waters Oasis HLB column $(25 \mathrm{~mm}, 2.1 \times 20 \mathrm{~mm}$, stationary phase: specific ratio of two monomers, hydrophilic Nvinylpyrrolidone and lipophilic divinylbenzene; Waters, Milford, USA) was used. First $30 \mu \mathrm{L}$ of derivatized sample extract was injected and loaded onto the extraction column in valve position A. The mobile phase was water/methanol 90/10 (v/v), delivered at a flow rate of $4 \mathrm{~mL} / \mathrm{min}$. Potentially interfering compounds were washed into waste. In parallel, the analytical column (Waters xBridge Shield RP18, $2.1 \times 150 \mathrm{~mm} ; 3.5 \mathrm{~mm}$ particle size) was equilibrated with $1 \mathrm{~g} / \mathrm{L}$ formic acid-acetonitrile $/ 10 \mathrm{mM}$ ammonium formiate $60 / 40$ (v/v), delivered at a flow rate of $0.4 \mathrm{~mL} / \mathrm{min}$. After $1 \mathrm{~min}$, the switching valve was changed to position $\mathrm{B}$; the extraction column was now eluted in a backflush mode onto the analytical column. After another $2 \mathrm{~min}$, the valve was switched back to position A. During the analytical chromatography into the mass spectrometer in position A, the extraction column was washed with acetonitrile/methanol 50/50 (v/v) at a flow rate of $4 \mathrm{~mL} /$ min for 1 min and the extraction column subsequently re-equilibrated with water/methanol 90/10 (v/v). The analytical column was kept at $38^{\circ} \mathrm{C}$ in a column oven, while the extraction column was kept at ambient temperature. 
itoring (MRM) trace of dibutyl-MMA and the internal standard dibutyl- $\mathrm{d}_{3}$-MMA were assessed. No fit weighting or axis transformation was applied.

An essential characteristic of quantification of MMA by LC-MS/MS is the need to obtain specificity towards succinic acid (SA) - which is isobaric with MMA and occurs naturally. While Kushnir et al. report that these isobaric compounds are distinguishable based on different fragmentation patterns, we were not able to obtain differentiation by MS/ MS. Consequently, baseline chromatographic separation of MMA from SA is required for reliable quantification of urinary MMA (molecular structures of MMA, SA and $\mathrm{d}_{3}$-MMA are shown in the data Supplement Figure 1). To verify complete chromatographic separation of dibutyl-MMA from dibutyl-SA, we analyzed pure solutions of the respective compounds as well as a mixture of both. We were able to demonstrate baseline separation and a representative MRMchromatogram is shown in Figure 1B.

To test the efficacy of the on-line solid phase extraction protocol, derivatized samples were injected directly onto the analytical column, and for comparison, onto the entire analytical system including the on-line solid phase extraction. This was performed in triplicate for the three quality control samples. We observed approximately 30\% higher MRM peak areas in the MRM trace of dibutyl- $\mathrm{d}_{3}$-MMA when applying on-line SPE compared to direct injection of the extracts (mean peak area of the QC samples without on-line SPE, 986 and using on-line SPE, 1271).

To study the recovery of the method, five urine samples were spiked with a pure solution of MMA $(50 \mu \mathrm{L}$ sample was spiked with $50 \mu \mathrm{L}$ of a $101.6 \mu \mathrm{mol} / \mathrm{L}$ MMA solution) prior to sample preparation. Comparative analyses of spiked vs. unspiked samples demonstrated a mean recovery rate of 95.4\% (range: $91.8 \%-97.9 \%$ ).

In order to characterize the sensitivity of the analytical system, a MMA solution with a concentration of $3.2 \mu \mathrm{mol} / \mathrm{L}$ was analyzed in triplicate. A mean signal-to-noise ratio of 36:1 was found.

Ion-suppression characteristics of the method were assessed by connecting a T-piece between the chromatographic column and the ion source. Using a syringe pump, a pure solution of dibutyl-MMA $(12.7 \mu \mathrm{mol} / \mathrm{L})$ was delivered at a constant flow rate $(0.2 \mathrm{~mL} / \mathrm{min})$ into the T-piece. With this post-column infusion, a baseline offset was generated in the MRM-trace of dibutyl-MMA. We did not observe any significant variation of this offset upon injection of human urine samples, ruling out relevant ion suppression by residual matrix components.

Potential carry over was excluded by injecting extracts from the highest quality control sample in quadruplicate, immediately followed by injection of a blank sample. The chromatogram for this sample showed no peaks in the MRM trace of dibutyl-MMA.

In four analytical series, applying full calibration, the mean observed results of the three quality control samples were compared with the expected concentrations and accuracy was calculated. We demonstrated mean accuracy and coefficients of variation below $10 \%$ (Table 1). Furthermore, we found that the coefficient of determination of the seven calibrator samples was $>0.99$ in all series.

In addition, inter-assay coefficients of variation were calculated for two different quality control samples which were analyzed over a six week period in 14 series for application of the method in an epidemiological study. These two pool samples showed a coefficient of variation of $8.2 \%$ (mean concentration $5.3 \mu \mathrm{mol} / \mathrm{L}$ ) and $5.2 \%$ (mean concentration $20.0 \mu \mathrm{mol} / \mathrm{L})$.

Apart from several articles which describe analysis of underivatized MMA by LC-MS/MS using electrospray ionisation in the negative mode $(12,13)$, the majority of published work uses the positive mode. This may be attributed to a rather low ion yield, using negative ionization in many instruments including ours. In order to use the positive ionisation mode, derivatization of MMA to dibutyl-MMA is required. SPE is used in the method presented here, primarily to protect the ion source from the aggressive derivatization mixture and second, in order to avoid the second evaporation step which was applied by Kushnir et al. (10) following the derivatization step. Our modified sample preparation protocol realizes a time saving of more than $1 \mathrm{~h}$ for a batch of 50 samples when compared to the method described by Kushnir et al.

We confirmed close agreement between the method described here and the method described by Kushnir et al. Ten samples analyzed by both methods showed a mean deviation of $2.2 \%$; range: $-9.6 \%$ to $+11.0 \%$.

The additional analytical run time required for the auxiliary on-line SPE sample clean-up step is moderate with 1 min (or approx. $15 \%$ of the run-time); only one additional isocratic pump and a switching valve is required. According to our extensive experience with quantification of immunosuppressants (14), on line SPE columns made of multifunc-

Table 1 Results of the method validation study (four-fold determination of spiked quality control samples in four analytical series).

\begin{tabular}{llllll}
\hline & $\begin{array}{l}\text { Target } \\
\text { concentration, } \\
\mu \text { mol/L }\end{array}$ & $\begin{array}{l}\text { Found } \\
\text { concentration } \\
(\text { mean}), \mu \mathrm{mol} / \mathrm{L}\end{array}$ & $\begin{array}{l}\text { Mean } \\
\text { accuracy } \\
(\mathrm{n}=16 \text { in } \\
4 \text { series }), \%\end{array}$ & $\begin{array}{l}\text { Total coefficient } \\
\text { of variation } \\
(\mathrm{n}=16 \text { in } \\
4 \text { series), } \%\end{array}$ & $\begin{array}{l}\text { Intra-assay } \\
\text { coefficient } \\
\text { of variation } \\
(\mathrm{n}=4 ; \text { range) }\end{array}$ \\
\hline QC level 1 & 29.4 & 28.9 & -2.0 & 8.2 & $5.3 \%-11.6 \%$ \\
QC level 2 & 80.2 & 73.2 & -8.7 & 5.5 & $2.3 \%-6.7 \%$ \\
QC level 3 & 173 & 159 & -8.3 & 6.0 & $3.0 \%-6.6 \%$ \\
\hline
\end{tabular}


tional co-polymer are very robust and can be used for several thousand injections.

The results of the method validation study (sensitivity, ion suppression effects, carry over, as well as imprecision and accuracy), qualify the assay described here as being suitable for routine clinical application. However, when considering that the method incorporates isotope-dilution internal standardisation, coefficients of variation above 5\% are surprising; which is in qualitative agreement with previous studies $(10,11)$. It may be assumed that isotope effects involved in the processes of derivatization, ionisation, and/or fragmentation could account for this observation. It has been shown previously that LC-MS/MS may be more prone to isotope effects than that observed in GC-MS (15).

The method was found to be convenient and robust with the approach of on-line SPE employing column switching in a large scale application: analysis of several hundred samples did not lead to any visible spoiling of the ion source components and no loss in sensitivity.

In summary, based on our results and experience, we can recommend the improved protocol described here. Our protocol involves the first on line SPE, and omits one evaporation step for the quantification of urinary MMA in the routine clinical laboratory setting.

\section{Acknowledgements}

This study was supported by the Hans-Fischer-Gesellschaft München.

\section{Conflict of interest statement}

Authors' conflict of interest disclosure: The authors stated that there are no conflicts of interest regarding the publication of this article. Research support played no role in the study design; in the collection, analysis, and interpretation of data; in the writing of the report; or in the decision to submit the report for publication.

Research funding: None declared.

Employment or leadership: None declared.

Honorarium: None declared.

\section{References}

1. Bolann BJ, Solli JD, Schneede J, Grøttum KA, Loraas A, Stokkeland M, et al. Evaluation of indicators of cobalamin deficiency defined as cobalamin-induced reduction in increased serum methylmalonic acid. Clin Chem 2000;46:1744-50.
2. Klee GG. Cobalamin and folate evaluation: measurement of methylmalonic acid and homocysteine vs vitamin $\mathrm{B}_{12}$ and folate. Clin Chem 2000;46:1277-83.

3. Stover PJ. Physiology of folate and vitamin B12 in health and disease. Nutr Rev 2004;62:S3-12.

4. Vogeser M, Seger C. A decade of HPLC-MS/MS in the routine clinical laboratory - goals for further developments. Clin Biochem 2008;41:649-62.

5. Lindgren A. Elevated serum methylmalonic acid. How much comes from cobalamin deficiency and how much comes from the kidneys? Scand J Clin Lab Invest 2002;62:15-20.

6. Vogiatzoglou A, Oulhaj A, Smith AD, Nurk E, Drevon CA, Ueland PM, et al. Determinants of plasma methylmalonic acid in a large population: implications for assessment of vitamin B12 status. Clin Chem 2009;55:2198-206.

7. Norman EJ. Urinary methylmalonic acid/creatine ratio defines true tissue cobalamin deficiency. Br J Haematol 1998;100: 605-18.

8. Rasmussen K, Nathan E. The clinical evaluation of cobalamin deficiency by determination of methylmalonic acid in serum or urine is not invalidated by the presence of heterozygous methylmalonic-acidaemia. J Clin Chem Clin Biochem 1990;28:419_ 21.

9. Norman EJ, Berry HK, Denton MD. Identification and quantitation of urinary dicarbocylic acids as their dicyclohexyl esters in disease states by gas chromatography mass spectrometry. Biomed Mass Spectrom 1979;6:546-53.

10. Kushnir MM, Komaromy-Hiller G, Shushan B, Urry FM, Roberts WL. Analysis of dicarboxylic acids by tandem mass spectrometry. High-throughput quantitative measurement of methylmalonic acid in serum, plasma, and urine. Clin Chem 2001;47:1993-2002.

11. Magera M, Helgeson JK, Matern D, Rinaldo P. Methylmalonic acid measured in plasma and urine by stable-isotope dilution and electrospray tandem mass spectrometry. Clin Chem 2000; 46:1804-10.

12. Blom HJ, van Rooij A, Hogeveen M. A simple high-throughput method for the determination of plasma methylmalonic acid by liquid chromatography-tandem mass spectrometry. Clin Chem Lab Med 2007;45:645-50.

13. Lakso HA, Appelblad P, Schneede J. Quantification of methylmalonic acid in human plasma with hydrophilic interaction liquid chromatography separation and mass spectrometric detection. Clin Chem 2008;54:2028-35.

14. Vogeser M, Fleischer C, Meiser B, Groetzner J, Spöhrer U, Seidel D. Quantification of sirolimus by liquid chromatography-tandem mass spectrometry using on-line solid-phase extraction. Clin Chem Lab Med 2002;40:40-5.

15. Lindegardh N, Annerberg A, White NJ, Day NP. Development and validation of a liquid chromatographic-tandem mass spectrometric method for determination of piperaquine in plasma stable isotope labeled internal standard does not always compensate for matrix effects. J Chromatogr B Analyt Technol Biomed Life Sci 2008;862:227-36. 